\title{
Ice iron/sodium film as cause for high noctilucent cloud radar reflectivity
}

\author{
P. M. Bellan ${ }^{1}$ \\ Received 6 February 2008; revised 30 April 2008; accepted 12 June 2008; published 26 August 2008.
}

[1] Noctilucent clouds, tiny cold electrically charged ice grains located at about $85 \mathrm{~km}$ altitude, exhibit anomalously high radar reflectivity. It is shown that this observed high radar reflectivity can be explained by assuming the ice grains are coated by a thin metal film; substantial evidence exists indicating that such a film exists and is caused by the deposition of iron and sodium atoms on the ice grain from iron and sodium layers located immediately above the noctilucent cloud layer. The number of conduction electrons in the thin metal film coating an ice grain is very large. When averaged over the volume occupied by a large number of ice grains, the quivering of these metal film electrons provides a much larger contribution to radar reflectivity than does the much smaller number of dusty plasma electrons or electron holes. Using observations indicating that noctilucent clouds are the dominant sink for the summer-time iron and sodium layers, it is shown that a sufficiently thick metal layer should form on a typical ice grain in a few hours to a few days.

Citation: Bellan, P. M. (2008), Ice iron/sodium film as cause for high noctilucent cloud radar reflectivity, J. Geophys. Res., 113, D16215, doi:10.1029/2008JD009927.

\section{Introduction}

\subsection{Context}

[2] Noctilucent clouds (also called polar mesospheric clouds) exist at $\sim 85 \mathrm{~km}$, an altitude much higher than ordinary clouds and have a vertical extent of $1-2 \mathrm{~km}$. Because of their many unusual properties noctilucent clouds have received considerable attention in recent years. It is now known that noctilucent clouds consist of tiny, extremely cold grains of water ice that can be either negatively or positively charged. Noctilucent clouds occur only when the ambient temperature is $\sim 150 \mathrm{~K}$, a situation that occurs at $85 \mathrm{~km}$ altitude in polar regions during the summer. A mysterious property of noctilucent clouds is that they exhibit anomalously large radar reflectivities [Havnes et al., 1992]; a nominal radar setup is sketched in Figure 1a. Since the ratio of grain radius $r_{d} \sim 10-100 \mathrm{~nm}$ to radar wavelength $\lambda \sim 1 \mathrm{~m}$ is $r_{d} / \lambda \sim 10^{-8}-10^{-7}$, Rayleigh scattering, which scales as $\left(r_{d} / \lambda\right)^{4}$ times the grain's geometric cross section, is much too small to explain the observed reflectivity. Various mechanisms have been proposed to explain the anomalously large reflectivity, but none have been compelling [Rapp et al., 2003b]. The purpose of this paper is to propose a new mechanism for why noctilucent clouds are such excellent radar reflectors. This mechanism is related to observations that noctilucent clouds are closely coupled to another strange phenomenon, high-altitude metal layers.

\footnotetext{
${ }^{1}$ Applied Physics, Caltech, Pasadena, California, USA.
}

Copyright 2008 by the American Geophysical Union. 0148-0227/08/2008JD009927\$09.00

\subsection{Brief Summary of Existing Models and Associated Issues}

[3] Complete radio wave reflection from a plasma occurs if the wave frequency is lower than the plasma frequency, i.e., if $f<f_{p e}=(2 \pi)^{-1} \sqrt{n e^{2} / \varepsilon_{0} m_{e}}$ where $n$ is the density of free electrons. A noctilucent cloud dust grain could be charged negatively because of ambient electron flux on the grain from ambient plasma or it could be charged positively because of photo-emission. The electron densities associated with both positive and negative charging are many orders of magnitude too small to explain the observed large radar reflectance. For example, reflection of an $f=50 \mathrm{MHz}$ radio wave at a layer having $f_{p e}=f$ would require an electron density $n=3 \times 10^{13} \mathrm{~m}^{-3}$ which is four orders of magnitude larger than the electron density at the relevant altitude. Thus reflection must be via some other means.

[4] Existing models proposed to explain radar echoes from noctilucent clouds are based on extensions of turbulent scattering theories developed previously in the context of weather radar. According to these models, the radar echo is caused by backscattering from isotropic spatial irregularities having a wavelength at the Bragg scale, i.e., a wavelength half that of the incident radar signal (for a $50 \mathrm{MHz}$ radar, the echo would therefore result from backscattering by spatial irregularities having a $3 \mathrm{~m}$ wavelength). Reviews of these models are given by Cho and Kelley [1993] and by Rapp and Lubken [2004].

[5] These existing models assume that the density irregularities are a consequence of isotropic neutral air turbulence whereby energy cascades from large to small scale eddies resulting in a characteristic partitioning of energy amongst the various scale subranges. In particular, the model assumes that large-scale eddies decay non-dissipatively to 
(a)

(b) (c)

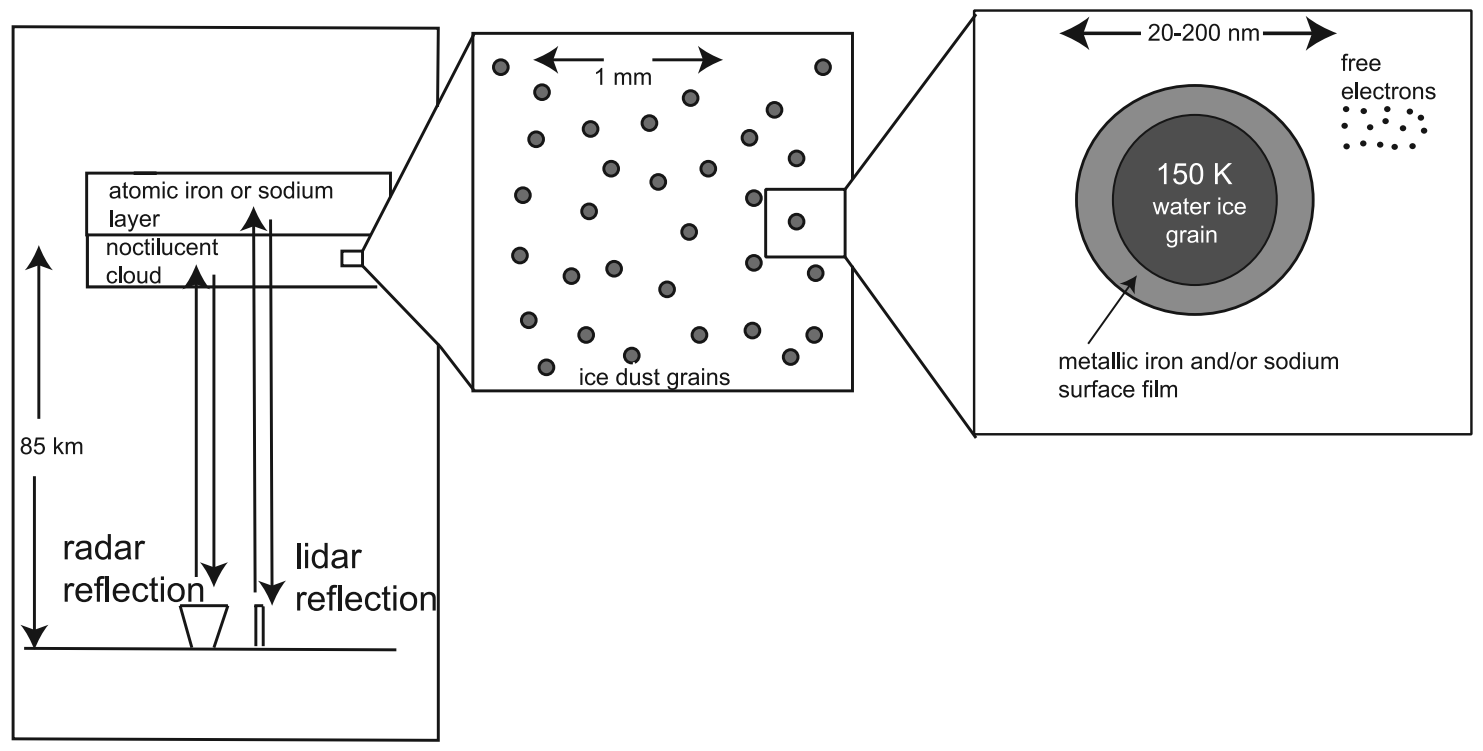

Figure 1. Sequence of inserts showing $\mathrm{Fe}$ or $\mathrm{Na}$ film coating noctilucent cloud water ice grains. Some representative free electrons external to the dust grain are also shown.

intermediate-scale eddies which in turn decay to still smaller eddies until at some ultimate small-scale viscous dissipation becomes important and the energy is transformed into heat. The long wavelength or non-dissipative subrange is called the inertial scale and has a $k^{-5 / 3}$ spectral power dependence. The short wavelength viscous subrange has a $k^{-p}$ spectral power dependence where $p$ is larger than $5 / 3$ and depends on the details of the dissipation mechanism.

[6] The $3 \mathrm{~m}$ Bragg wavelength at which $50 \mathrm{MHz}$ radar echoes are produced is well into the viscous subrange. This produces a severe difficulty for the existing model because the spectral energy of isotropic neutral air turbulence in the viscous subrange is orders of magnitude too small to explain the strength of the radar reflection [Cho and Kelley, 1993; Rapp et al., 2003b]. In order to boost spectral energy at the $3 \mathrm{~m}$ wavelength, effort has gone into modifying the existing model by arguing that dust grains being heavy, diffuse much less than air molecules, so collections of dust grains would have long-lived spatial irregularities with $3 \mathrm{~m}$ wavelength [Cho and Kelley, 1993; Rapp et al., 2003b]. This means that the dust is no longer a tracer of the air turbulence, but instead follows its own dynamics, and furthermore implies that the radar is somehow scattering off the pattern of the dust rather than the pattern of the air.

[7] A related issue results from the fundamental fact that reflection of an electromagnetic wave results from the wave accelerating electrons which then re-radiate. This means that Bragg reflection of a radar pulse must involve spatial irregularities of electron density which are somehow coupled to spatial irregularities of dust density. However, measurements by rockets traversing noctilucent clouds often show electron "bite-out" [Rapp et al., 2003a], a complete depletion of electrons at the altitude where maximum radar reflection takes place. Rapp et al. summarize this issue by stating "the natural question arises why we observe a radar signal from altitudes where the in situ measured electron number density is zero." Evidence in support of existing models is thus mixed and so the question of what provides the reflection mechanism remains open.

\section{Metallic Coating of Water Ice Grains}

[8] Noctilucent clouds have a close association with narrow high-altitude layers of atomic $\mathrm{Na}, \mathrm{K}, \mathrm{Ca}$, or $\mathrm{Fe}$ vapors [Plane, 2003; Plane et al., 2004; Raizada et al., 2007]. This relationship is sketched in Figure 1a; the iron metal layer is the most significant as it has the highest number density [Plane, 2003], while the sodium layer is the next most significant being about a factor of 2-10 smaller. The Na thermal velocity is $\sim 50 \%$ larger than that of $\mathrm{Fe}$, so the $\mathrm{Na}$ flux could be significant even if the $\mathrm{Na}$ density is smaller. Because of the larger $\mathrm{Na}$ thermal velocity, because $\mathrm{Na}$ layers have been more widely studied, and because $\mathrm{Na}$ is more important for photo-emission, both $\mathrm{Fe}$ and $\mathrm{Na}$ will be considered here while realizing that most of the time Fe is dominant. The properties of these atomic metal vapor layers have been measured using LIDARs (pulsed lasers tuned to resonate with optical lines of specific metal atoms) as sketched in Figure 1a. The radar-like ranging LIDAR echo gives a measurement of atomic number density versus altitude. Consistent with standard usage, here a 'metal layer' means a layer of atomic vapor and should not be confused with a solid metal. The atoms comprising the metal layer come from the ablation of a continuous flux of micrometeoroids impinging on the Earth [Plane, 2003; Plane et $a l ., 2004]$. The distinct heights of metal layers of different atomic species result from a differential ablation (fractionation) process whereby a metal with lower melting/evaporation temperatures ablates before a metal with higher melting/evaporation temperatures [McNeil et al., 1998]. The ablation process depletes the entire inventory of the most volatile micrometeoroid constituents, $\mathrm{Na}$ and $\mathrm{K}$. Hoffner and Friedman [2004] have shown that the relative 
abundances of $\mathrm{Na}, \mathrm{K}, \mathrm{Ca}$, and $\mathrm{Fe}$ in atmospheric metal layers are constant over time. A close relationship exists between noctilucent clouds and the $\mathrm{Fe}, \mathrm{Na}$, and $\mathrm{K}$ metal layers. In particular, a metal layer is always located immediately above a cloud layer [e.g., see Lubken and Hoffner, 2004, Figure 3] and it appears that the underside of the metal layer is being depleted by the top side of the cloud layer [Plane et al., 2004].

[9] Although typically negatively charged, noctilucent cloud grains have also been observed to become positively charged on occasion [Havnes et al., 1996; Smiley et al., 2006], a property attributed to photo-emission of electrons from the noctilucent cloud grains. Havnes and Naesheim [2007] have recently questioned the validity of the Havnes et al. [1992] measurement because of a confounding effect due to secondary electron emission in the detector, but there does not seem to be any question about the validity of the Smiley et al. measurements of positively charged grains since these measurements took into account grain trajectories.

[10] Eidhammer and Havnes [2001] argued that the $8.7 \mathrm{eV}$ work function of pure ice is much too large for any significant photoemission to occur from pure-ice noctilucent cloud grains because the solar photon flux at $8.7 \mathrm{eV}$ and above is negligible. Since there is evidence that noctilucent cloud grains can become positively charged, Eidhammer and Havnes proposed that the grain surface might be contaminated with a material having a much lower work function than pure ice. Rapp and Lubken [1999] suggested that the photoemission of noctilucent cloud grains required for the observed occasional positive charging could be explained if the grain were solid metallic $\mathrm{Na}\left(W_{w f}=\right.$ $2.28 \mathrm{eV}$ ) while Havnes et al. [1992], Murray and Plane [2005] suggested that only the surface of the grain need be Na. Vondrak et al. [2006a, 2006b] have recently reported measurements from a controlled laboratory experiment showing that water ice with less than a monolayer of $\mathrm{Na}$ has a workfunction lowered to $2.5 \mathrm{eV}$, a rather dramatic effect. This suggests that the $\mathrm{Na}$ bound to the ice is behaving in a metallic fashion, i.e., has highly mobile electrons. Iron has a higher work function $\left(W_{w f}^{F e}=4.7 \mathrm{eV}\right)$ but this is still much lower than pure ice so photo-emission would be expected to occur if the ice grain were coated with iron or with a combination of iron and sodium.

[11] Coating of noctilucent cloud dust grains by a thin metallic film is thus quite likely because:

[12] 1. An extremely strong correlation exists between the altitude at which atmospheric atomic $\mathrm{Fe}$ and $\mathrm{Na}$ layers stop and noctilucent clouds start as sketched in Figure 1a suggesting that the extraterrestrial influx of $\mathrm{Fe}$ and $\mathrm{Na}$ is completely absorbed on the surface of noctilucent clouds [see Plane et al., 2004; Murray and Plane, 2005].

[13] 2. Iron and sodium are continuously deposited in Earth's upper atmosphere by micrometeoroids [Plane, 2003; Plane et al., 2004; Murray and Plane, 2005].

[14] 3. Cold $(<150 \mathrm{~K})$ noctilucent ice grains manifest a rapid and complete uptake of any incident $\mathrm{Fe}$ or $\mathrm{Na}$ vapor flux [Plane et al., 2004; Gardner et al., 2005]. A substantial depletion of mesospheric $\mathrm{Fe}$ and $\mathrm{Na}$ metal atoms is observed when a noctilucent cloud is present and the underside of the $\mathrm{Fe}$ and $\mathrm{Na}$ layers have a very steep-scale height [Plane, 2003; Plane et al., 2004; Raizada et al., 2007; Fan et al., 2007] at the altitude where the cloud begins.
[15] 4. Yakshinskiy and Madey [2001] examined deposition of thin films of alkali metals on ice at the relevant low temperatures. Using photoelectron spectroscopy they showed that the first monolayer of deposited potassium is not metallic, but then subsequent layers are metallic. Most recently Plane et al. [2004] and Murray and Plane [2005] have shown using carefully designed laboratory experiments that a rapid and essentially complete uptake of Fe and $\mathrm{Na}$ vapor by ice occurs in the temperature range of interest and that this uptake results in the deposition of a thin film of solid metallic $\mathrm{Fe}$ or $\mathrm{Na}$ on the surface of the ice.

[16] There is thus a strong tendency for vapor-phase atomic $\mathrm{Fe}$ and $\mathrm{Na}$ to condense on $150 \mathrm{~K}$ ice grains and form a solid metallic film. Since there is a continuous extraterrestrial micrometeoroid-borne flux of $\mathrm{Fe}$ and $\mathrm{Na}$, the $150 \mathrm{~K}$ noctilucent ice grains should easily become coated with a thin metallic $\mathrm{Fe}$ and/or $\mathrm{Na}$ film by the uptake of the $\mathrm{Fe} / \mathrm{Na}$ atoms ablating from micrometeoroids. Coatings of other metals (e.g., K) could also develop, depending on the ablation details. Coating of noctilucent cloud ice grains is sketched in Figures $1 \mathrm{~b}$ and $1 \mathrm{c}$.

\section{Proposed Mechanism for Anomalous Radar Reflectance}

[17] We begin the presentation of our model with a reexamination of the basic mechanism responsible for the back-scattering of an electromagnetic wave. Back-scattering is ultimately due to coherent electromagnetic radiation by an oscillating electric current. This current is composed of electrons accelerated by the incident wave so as to quiver with just the right phase and polarization to excite the backscattered wave. Our proposed mechanism involves this same electron quivering but the summation of the contributions of individual electrons to form a macroscopic current will involve heterogenous considerations rather than the usual assumption of fluid-like microscopic electron homogeneity and also the electrons might not be completely free.

[18] The propagation of electromagnetic waves in a plasma is governed by Ampere's and Faraday's laws

$$
\begin{gathered}
\nabla \times \mathbf{B}=\mu_{0} \mathbf{J}+\mu_{0} \varepsilon_{0} \frac{\partial \mathbf{E}}{\partial t} \\
\nabla \times \mathbf{E}=-\frac{\partial \mathbf{B}}{\partial t}
\end{gathered}
$$

where the wave electric field is given by

$$
\mathbf{E}(\mathbf{x}, t)=\tilde{\mathbf{E}}(\mathbf{x}) \exp (-i \omega t)
$$

We will consider the possibility that the electrons are either (1) free or (2) metallic in which case they have a collision frequency $v_{\text {metal }}$. Both situations (1) and (2) can be described using the electron equation of motion

$$
m_{e} \frac{d \mathbf{v}}{d t}=-e \mathbf{E}(\mathbf{x}, t)-v m_{e} \mathbf{v}
$$


(a)
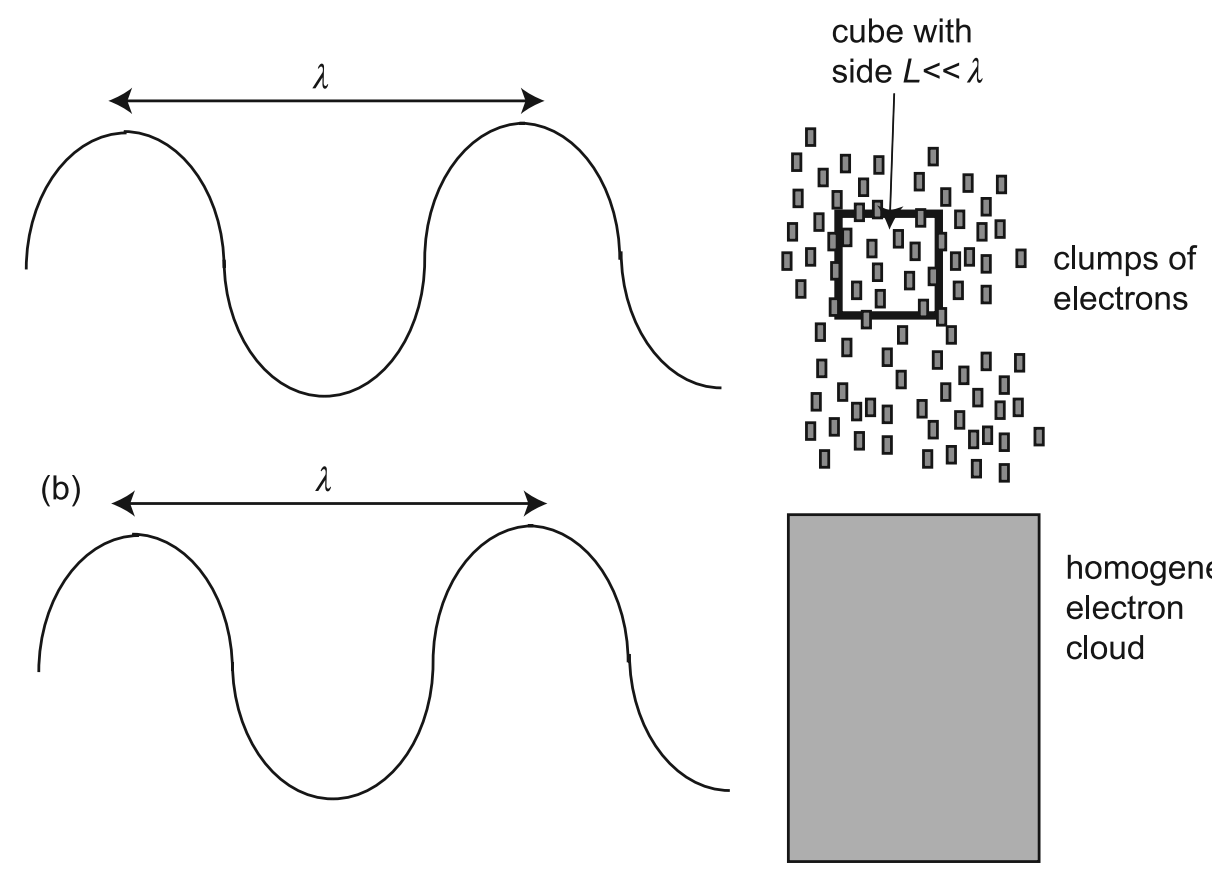

homogeneous

electron

cloud

Figure 2. (a) Electromagnetic wave reflecting from a clumpy distribution of free electrons. (b) Electromagnetic wave reflecting from a homogeneous distribution of free electrons having the same density as defined by equation (7). It is argued that the wave will reflect in the same manner for both electron distributions.

which has the solution

$$
\mathbf{v}_{e, q u i v e r}=\frac{e \tilde{\mathbf{E}}(\mathbf{x})}{m_{e}(i \omega-v)} \exp (-i \omega t)
$$

The current $\mathbf{J}$ is given by

$$
\mathbf{J}=-n_{e, q u i v e r} \mathbf{v}_{e, q u i v e r}=-\frac{n_{e, q u i v e r} e^{2} \tilde{\mathbf{E}}(\mathbf{x})}{m_{e}(i \omega-v)} \exp (-i \omega t)
$$

where $n_{e, q u i v e r}$ is the density of the quivering electrons. A critical aspect of our model is that we will use a less constrained definition than convention for the density of the quivering electrons. In particular, we define the density of the quivering electrons to be

$$
n_{e, \text { quiver }}=\lim _{l \ll L \ll \lambda} \frac{N_{\text {quiver }}(L)}{L^{3}}
$$

where $N_{\text {quiver }}(L)$ is the number of quivering electrons in a cube of volume $L^{3}, \lambda$ is the wavelength of the incident electromagnetic wave, and $l=n_{d}^{-1 / 3}$ is the nominal distance between dust grains. This situation is sketched in Figure 2a which contrasts with the conventional situation shown in Figure $2 \mathrm{~b}$. Because a wave cannot resolve irregularities smaller than its own wavelength, the actual value of $L$ is unimportant if $L \ll \lambda$ provided a large number of quivering electrons exist within a cube of volume $L^{3}$. Furthermore, if $L \ll \lambda$, quivering electrons inside the cube of volume $L^{3}$ quiver in phase with each other so their spatial distribution within the cube is of no consequence. Thus the electron distribution within a cube $L^{3}$ need not be homogeneous provided $L \ll \lambda$. The clumpy free electrons sketched in Figure $2 \mathrm{a}$ will interact with an incident electromagnetic wave in exactly the same way as the spatially homogeneous electron distribution shown in Figure $2 \mathrm{~b}$ provided both the clumpy and homogeneous electrons have the same density as defined by equation (7).

[19] We now estimate the effective value of $n_{e, \text { quiver }}$ for noctilucent cloud ice grains coated by a thin metallic $\mathrm{Fe}$ and/or Na film. Wood [1933] noted that metallic $\mathrm{Na}$ becomes transparent at wavelengths shorter than $\lambda_{\text {cutoff }}=$ $210 \mathrm{~nm}$; this cutoff wavelength indicates that metallic $\mathrm{Na}$ has an electron plasma frequency $f_{p e}^{N a}=c / \lambda_{\text {cutoff }}=1.43 \times$ $10^{15} \mathrm{~Hz}$. This electron plasma frequency implies that the density of conduction electrons in the Na metal is

$$
n_{e, \text { metal }}=\left(2 \pi f_{p e}^{N a}\right)^{2} \varepsilon_{0} m / e^{2}=2.5 \times 10^{28} \mathrm{~m}^{-3} .
$$

Fedorov et al. [2007] report the plasma frequency of thin films of $\mathrm{Fe}$ to be $f_{p e}^{\mathrm{Fe}} \simeq 1.6 \times 10^{15} \mathrm{~Hz}$ implying approximately the same density of conduction electrons as for Na; thus we will assume $n_{e, \text { metal }}=2.5 \times 10^{28} \mathrm{~m}^{-3}$ for both $\mathrm{Fe}$ and $\mathrm{Na}$.

[20] The electrical resistivity of metallic $\mathrm{Na}$ at $150 \mathrm{~K}$ is $\eta=2 \times 10^{-8}$ Ohm-m [Bradshaw and Pearson, 1956], while the electrical resistivity of metallic $\mathrm{Fe}$ at $150 \mathrm{~K}$ is $\eta=3 \times 10^{-8} \mathrm{Ohm}-\mathrm{m}$. In order to consider both $\mathrm{Fe}$ and $\mathrm{Na}$, we will assume that $\eta \sim 2.5 \times 10^{-8} \mathrm{Ohm}-\mathrm{m}$ to approximate both with reasonable accuracy. The effective electron collision frequency is then $v_{\text {metal }}=\eta n_{e, \text { metal }} e^{2} / m_{e}=$ $1.8 \times 10^{13} \mathrm{~s}^{-1}$ which is four to six orders of magnitude 
higher than the 50-1000 MHz radar frequencies used. The radar waves will penetrate a collisional skin depth $\delta=$ $\sqrt{\eta / \mu_{0} \omega}$ into the metallic film; at $50 \mathrm{MHz} \delta=8 \mu \mathrm{m}$ while at $1000 \mathrm{MHz} \delta=1.8 \mu \mathrm{m}$. Since the ice grains have radii $r_{d} \sim$ $10-100 \mathrm{~nm}$, any metallic Fe or $\mathrm{Na}$ film will be much thinner than the collisional skin depth in the 50-1000 MHz frequency range. The radar wave electric field will thus fully penetrate the metal film and so all electrons in the metal film will quiver in the wave electric field according to equation (5).

[21] A metal $\mathrm{Fe}$ or $\mathrm{Na}$ film of thickness $d$ coating an ice dust grain has volume $V_{\text {shell }}=4 \pi r_{d}^{2} d$. We now examine the effect the electrons in this spherical metal shell have on a noctilucent cloud constituted by ice dust grains having density $n_{d}$. The volumetric fill factor of metallic $\mathrm{Fe}$ or $\mathrm{Na}$ electrons quivering in response to the radar wave is

$$
n_{d} V_{\text {shell }}=4 \pi n_{d} r_{d}^{2} d
$$

This is the factor by which the density of the Fe or Na metal electrons quivering in the radar electric field is diluted when averaged over a cube of side dimension $L$ that is large compared to the $l=n_{d}^{-1 / 3}$ intergrain separation, but small compared to the radar wavelength $\lambda$. For example, if $n_{d}$ $\sim 10^{7} \mathrm{~m}^{-3}$ so $l \sim 5 \mathrm{~mm}$, one could choose $L=20 \mathrm{~mm}$ as the nominal size of the cube used for calculating effective electron density; $L=20 \mathrm{~mm}$ is microscopic compared to the radar wavelength which lies in the range $10^{-1} \mathrm{~m}<\lambda<$ $10^{1} \mathrm{~m}$. There would be $n_{d} L^{3} \sim 10^{2}$ dust grains in a cube of volume $L^{3}$.

[22] The spacing between metal film atoms is $n_{e, \text { metal }}^{-1 / 3}=$ $\left(2.5 \times 10^{28}\right)^{-1 / 3}=0.3 \mathrm{~nm}$. A metallic film coating the ice grain must be at least a monolayer thick in order to exhibit electrical conductivity and behave like a classic metal (see measurements by Yakshinskiy and Madey [2001]). If the deposition of metal on an ice grain is spatially nonuniform (splotchy), the deposited metal may still behave as a classic metal even if its average thickness is less than a monolayer.

[23] The effective density of metal electrons quivering in response to the radar wave, i.e., the density obtained using equation (7) where $l \ll L \ll \lambda$, is thus

$$
n_{e, \text { quiver }}^{\text {metal }}=n_{e, \text { metal }} n_{d} V_{\text {shell }}=4 \pi n_{e, \text { metal }} n_{d} r_{d}^{2} d
$$

[24] This can be compared with the density of free electrons (or electron holes due to depletion of electrons by absorption onto a dust grain) associated with charging of a dust grain; representative external electrons are sketched in Figure 1c.

[25] Smiley et al. [2006] reported sounding rocket measurements of ions, electrons, and charged dust grains at noctilucent cloud altitudes. The dust grains had various sizes and certain groups were positively charged while others were negatively charged. The positive charge was attributed to photo-emission of electrons by a dust grain in the manner reported by Murray and Plane [2005]. Particles with $30 \mathrm{~nm}$ radius were assumed to have an average charge $Z=-2$ consistent with the predictions of Rapp and Lubken [2001].

[26] The density of free electrons (or electron holes) associated with dust charging that can quiver in the wave is

$$
n_{e, q u i v e r}^{\text {free }}=n_{d} Z
$$

and $n_{e, q u i v e r}^{\text {free }}$ could at most be $3 \times 10^{9} \mathrm{~m}^{-3}$ based on the maximum free electron density measured at altitudes where there are significant dust grain densities (see $82.5 \mathrm{~km}$ altitude data of Figure 6 of Smiley et al. [2006]).

[27] The electrostatic potential of a dust grain having $Z$ charges is

$$
U=\frac{Z e}{4 \pi \varepsilon_{0} r_{d}}
$$

Rapp and Lubken [2001] have calculated $Z$ for noctilucent cloud dust grains and found that $Z=-3$ for $r_{d}=50 \mathrm{~nm}$ and that $Z$ scales linearly with $r_{d}$ for $r_{d}>10 \mathrm{~nm}$ (see the top of Figure 4 of Rapp and Lubken [2001]). Equation (12) shows that this corresponds to a grain charge $U=-0.086 \mathrm{~V}$. The actual value of $r_{d}$ is not well established and probably varies within the cloud, but is believed to lie between 10 and $100 \mathrm{~nm}$. For example, Plane [2004] argued that $r_{d}$ should lie between 50 and $100 \mathrm{~nm}$ in order to have realistic mixing ratios.

[28] Let us now calculate how the quivering electrons (either metal or free) contribute to wave back-scattering. Combination of Ampere's law and Faraday's law gives the usual wave equation

$$
\nabla \times \nabla \times \mathbf{E}=-\frac{\partial}{\partial t}\left(\mu_{0} \mathbf{J}+\mu_{0} \varepsilon_{0} \frac{\partial \mathbf{E}}{\partial t}\right)
$$

Considering transverse waves having $\nabla \cdot \mathbf{E}=0$ and using equation (6) this becomes

$$
\nabla^{2} \tilde{\mathbf{E}}(\mathbf{x})+k^{2} \tilde{\mathbf{E}}(\mathbf{x})-i \omega \frac{\mu_{0} n_{e, q u i v e r} e^{2} \tilde{\mathbf{E}}(\mathbf{x})}{m_{e}(i \omega-v)}=0
$$

where $k=\omega / c$. Let us assume that $\tilde{\mathbf{E}}(\mathbf{x})=\tilde{E}_{y}(z) \hat{y}$ and that propagation is in the $z$ direction so equation (14) reduces to

$$
\frac{d^{2} \tilde{E}_{y}}{d z^{2}}+k^{2} \tilde{E}_{y}-\frac{i \omega}{c^{2}} \frac{n_{e, q u i v e r}(z) e^{2}}{m_{e} \varepsilon_{0}(i \omega-v)} \tilde{E}_{y}=0 .
$$

Equation (15) reverts to the usual plasma wave dispersion $\omega^{2}=\omega_{p e}^{2}+K^{2} c^{2}$ in the limit $\omega \gg v$ as would be the case for free electrons external to the grain; equation (15) reverts to the collisional skin depth characterization in the opposite limit as would be the case for radio waves in a metal.

[29] We solve equation (15) using a perturbation technique based on the assumption that the scattered wave is weak. In particular, we assume

$$
E_{y}=\tilde{E}_{y 0}+\tilde{E}_{y 1}
$$

where $\tilde{E}_{y 0}=E_{0} \exp (i k z)$ and $\left|\tilde{E}_{y 1}\right| \ll E_{0}$. Substitution of equation (16) into equation (15) and dropping second and higher order terms gives

$$
\frac{d^{2} \tilde{E}_{y 1}}{d z^{2}}+k^{2} \tilde{E}_{y 1}=f(z)
$$


where

$$
f(z)=\frac{i \omega}{c^{2}} \frac{n_{e, q u i v e r}(z) e^{2}}{m_{e} \varepsilon_{0}(i \omega-v)} E_{0} \exp (i k z)
$$

is a source function. For convenience we now choose the $z$ origin to be at the cloud location in which case the radar is located at a large, negative $z$ while points above the cloud will have positive $z$. The boundary conditions are then (1) there can be both forward and reflected waves for large negative $z$, but (2) for large positive $z$ there can only be a forward wave (i.e., above the cloud there can only be an upward propagating wave).

[30] Equation (17) has the exact solution

$$
\tilde{E}_{y 1}(z)=\frac{1}{2 i k}\left(\begin{array}{c}
\exp (i k z) \int_{-\infty}^{z} f\left(z^{\prime}\right) \exp \left(-i k z^{\prime}\right) d z^{\prime} \\
-\exp (-i k z) \int_{+\infty}^{z} f\left(z^{\prime}\right) \exp \left(i k z^{\prime}\right) d z^{\prime}
\end{array}\right)
$$

where the lower limits of the integrals have been chosen to satisfy the boundary conditions that (1) $\tilde{E}_{y 1}$ cannot have a term of the form $\exp (-i k z)$ for $z \rightarrow \infty$ (no reflected wave above the cloud) and (2) $\tilde{E}_{y 1}$ cannot have a term of the form $\exp (+i k z)$ for $z \rightarrow-\infty$ (all the transmitted wave is accounted for by $\left.\tilde{E}_{y 0}\right)$.

[31] Since the radar is located at large negative $z$, the reflected wave seen by the radar receiver is

$$
\begin{aligned}
\tilde{E}_{\text {reffect }}(z) & =\lim _{z \ll 0} \tilde{E}_{y 1}(z) \\
& \simeq-\frac{1}{2 i k} \exp (-i k z) \int_{+\infty}^{-\infty} f\left(z^{\prime}\right) \exp \left(i k z^{\prime}\right) d z^{\prime} \\
& =-\frac{E_{0}}{2 c} \frac{n_{e, q u i v e r}(0) e^{2}}{(i \omega-v) m_{e} \varepsilon_{0}} \exp (-i k z) \int_{-\infty}^{\infty} g\left(z^{\prime}\right) \exp \left(2 i k z^{\prime}\right) d z^{\prime}
\end{aligned}
$$

where

$$
g(z)=\frac{n_{e, \text { quiver }}(z)}{n_{e, \text { quiver }}(0)}
$$

is the vertical profile of the quivering electrons.

[32] The fractional reflected power is

$$
\begin{aligned}
R & =\left|\tilde{E}_{\text {reflect }}(z)\right|^{2} / E_{0}^{2} \\
& =\frac{1}{4 c^{2}}\left(\frac{n_{e, \text { quiver }}(0) e^{2}}{m_{e} \varepsilon_{0}}\right)^{2}\left|\frac{1}{i \omega-v}\right|^{2}\left|\int_{-\infty}^{\infty} g\left(z^{\prime}\right) \exp \left(2 i k z^{\prime}\right) d z^{\prime}\right|^{2}
\end{aligned}
$$

which is consistent with Bragg scattering by fluctuations at half the wavelength of the wave.

[33] There are three possible sources for $n_{\text {equiver }}$ The first would be electrons in pre-existing plasma in which the dust grains happened to be immersed; the dust grains would scavenge some or all of these electrons so that the electron density profile would result from the remnants of whatever electrons were not scavenged. In this case the dust and electron densities would be anti-correlated; anti-correlation of dust and electron density fluctuations has been seen in rocket data for regions away from bite-out [Rapp et al., 2003a]. Anti-correlation of electrons and dust would not alter equation (22) because $1-g(z)$ has the same spectrum as $g(z)$. As Rapp et al. noted, if the dust grains scavenge all the free electrons (i.e., bite-out), there would be no free electrons left to quiver. The second possible source would be free electrons emitted when a dust grain becomes charged via photoemission of these electrons (i.e., the electrons at $82.5 \mathrm{~km}$ altitude in Figure 6a of Smiley et al. [2006]). In this case, electrons would have to be located near the dust grains in order to maintain quasi-neutrality and so the dust and the electron fluctuations would have the same profile $g(z)$. Because positive charging of dust grains is only rarely observed this situation is probably uncommon. The third possible source would be conduction electrons in the $\mathrm{Fe} / \mathrm{Na}$ metal film coating a dust grain. At radar frequencies, free electrons would have $v_{\text {free }} \ll \omega$ whereas conduction electrons in the metal film would have $v_{\text {metal }} \gg \omega$.

[34] Using equation (22) it is seen that the ratio of reflectance by metal electrons to the reflectance by free electrons is

$$
\frac{R_{\text {metal }}}{R_{\text {free }}}=\left(\frac{\omega n_{e, q u i v e r}^{\text {metal }}}{v_{\text {metal }} n_{e, \text { quiver }}}\right)^{2} .
$$

The contribution to scattering from the metallic film electrons will thus be more important than the contribution from free electrons if

$$
n_{e, \text { quiver }}^{\text {metal }}>\frac{v_{\text {metal }}}{\omega} n_{e, \text { quiver }}^{\text {free }} .
$$

[35] Substituting equations (10), (11), and (12) this can be expressed as

$$
d>\frac{v_{\text {metal }} \varepsilon_{0}|U|}{\omega n_{e, \text { metal }} e r_{d}} .
$$

Equation (25) applies if there are free electrons with profile $g(z)$ or electron holes with effective density corresponding to the negative of the density of the electrons scavenged by the dust grains. The hole argument would require the interstices between dust grains to be occupied by a conventional electron-ion plasma; measurements reported by Rapp et al. [2003a] showing anti-correlation between electron and dust fluctuations in regions not having complete bite-out would correspond to this situation of free electrons in the space between dust grains. However, if all available electrons are scavenged (i.e., bite-out), then equation (25) is too conservative because the right hand side of equation (24) would be zero and the only electrons available for reflecting the radar wave would be the conduction electrons in the metal film coating the dust grain.

[36] As a conservative estimate, let us assume that biteout has not occurred so there are electron holes, with effective density $n_{e, \text { quiver }}^{\text {free }}(z)=-Z_{d} n_{d}(z)$ in which case equation (25) provides the relevant threshold for when metal film electrons dominate. Using $v_{\text {metal }}=1.8 \times 10^{13} \mathrm{~s}^{-1}$, $n_{e, \text { metal }}=2.5 \times 10^{28} \mathrm{~m}^{-3}, f=\omega / 2 \pi=50 \mathrm{MHz}, U=-0.086 \mathrm{~V}$, and assuming $r_{d}=100 \mathrm{~nm}$, it is seen that metal film electrons dominate if $d>0.1 \mathrm{~nm}$, i.e., the metal film is on 


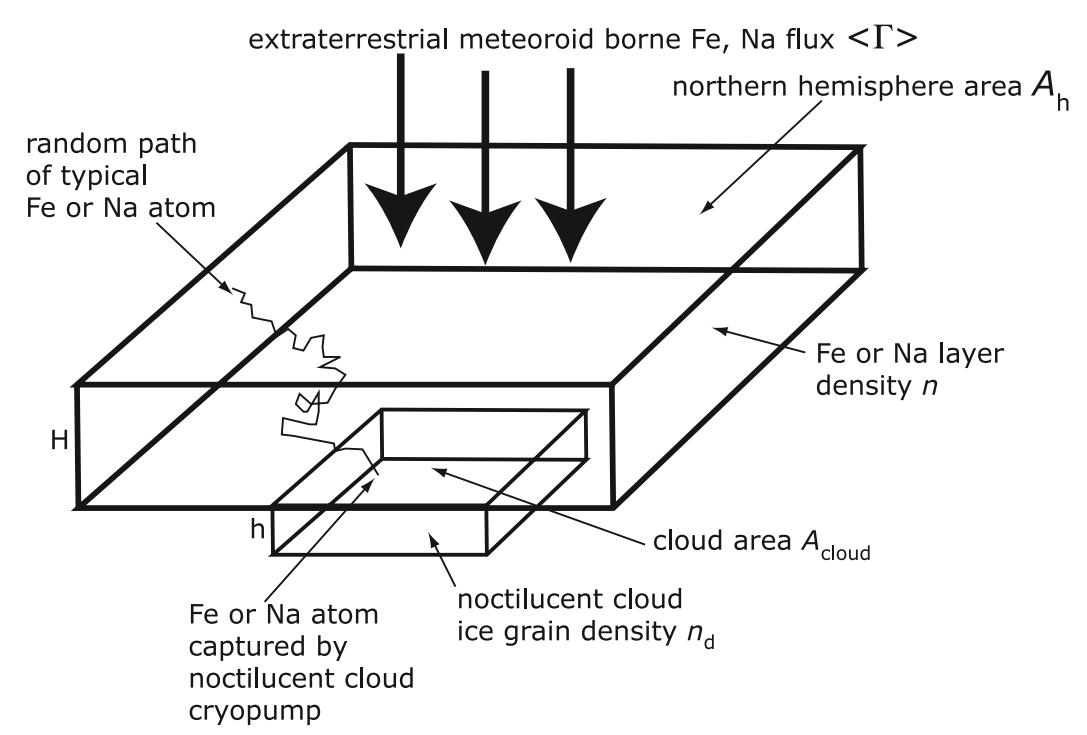

Figure 3. Schematic of summer-time flow $\mathrm{Fe} / \mathrm{Na}$ flow pattern wherein noctilucent cloud at the bottom of $\mathrm{Fe} / \mathrm{Na}$ layer acts as a sink for nearly all $\mathrm{Fe} / \mathrm{Na}$ atoms incident on the top of the $\mathrm{Fe} / \mathrm{Na}$ layer.

average about 0.3 monolayers thick. If $r_{d}=10 \mathrm{~nm}$ is assumed instead, the metal film electrons would dominate if $d>1 \mathrm{~nm}$. Equation (25) no longer makes sense when $d$ exceeds $r_{d}$ which would occur for $r_{d}<3 \mathrm{~nm}$; thus the model can be considered as self-consistent for $r_{d}$ much greater than $3 \mathrm{~nm}$, e.g., $r_{d}>10 \mathrm{~nm}$. Equation (25) also shows that scattering from the metal film becomes even more dominant as the radar wave frequency $\omega$ is increased.

[37] If metal electrons dominate, then equation (10) is used to give $n_{e, \text { quiver }}$ in which case equation (22) becomes

$$
R \simeq \frac{1}{4 c^{2}}\left(\frac{4 \pi n_{e, \text { metal }} n_{d}(0) r_{d}^{2} e^{2} d}{m_{e} \varepsilon_{0} v_{\text {metal }}}\right)^{2}\left|\int_{-\infty}^{\infty} g\left(z^{\prime}\right) \exp \left(2 i k z^{\prime}\right) d z^{\prime}\right|^{2}
$$

The scattered power at a radar frequency $f$ is thus essentially a measure of the spatial spectral density of the noctilucent cloud vertical profile at the wavenumber $2 k$. The observed extreme drop in reflected power at $1 \mathrm{GHz}$ compared to $50 \mathrm{MHz}$ [Rapp and Lubken, 2004] would then simply be an indication that the cloud profile has much more structure with characteristic length $3 \mathrm{~m}$ than with characteristic length $0.15 \mathrm{~m}$.

[38] If we assume that $g(z)$ is only finite in the range $-h / 2$ $<z<h / 2$ where $h$ is the noctilucent cloud vertical height, the "average power" associated with $g(z)$ can be defined as

$$
\begin{aligned}
\langle W\rangle & =\frac{1}{h} \int_{-h / 2}^{h / 2}[g(z)]^{2} d z \\
& =\frac{1}{h} \int_{-h / 2}^{h / 2}\left[\frac{1}{2 \pi} \int_{-\infty}^{\infty} G(k) e^{-i k z} d k\right]\left[\frac{1}{2 \pi} \int_{-\infty}^{\infty} G\left(k^{\prime}\right) e^{-i k^{\prime} z} d k^{\prime}\right] d z \\
& =\frac{1}{2 \pi h} \int_{-\infty}^{\infty} d k \int_{-\infty}^{\infty} d k^{\prime} G(k) G\left(k^{\prime}\right) \delta\left(k+k^{\prime}\right) \\
& =\frac{1}{2 \pi h} \int_{-\infty}^{\infty} d k G(k) G(-k) \\
& =\frac{1}{\pi h} \int_{0}^{\infty} d k G(k) G(-k)
\end{aligned}
$$

where

$$
G(k)=\int_{-\infty}^{\infty} g(z) \exp (i k z) d z
$$

is the Fourier transform of $g(z)$. Although by definition $g(z)$ vanishes outside the range $-h / 2<z<h / 2$, the limits of the integral in equation (28) have been extended to infinity to be in conformity with convention. Because $g(z)$ is real, $g(-k)$ is the complex conjugate of $g(k)$ and so we can define

$$
S(k)=\frac{1}{\pi h} G(k) G(-k)=\frac{1}{\pi h}\left|\int_{-\infty}^{\infty} g(z) \exp (i k z) d z\right|^{2}
$$

as the suitably normalized spectral power so that

$$
W=\int_{0}^{\infty} d k S(k)
$$

Keeping only the contribution $\tilde{n}_{e, B r a g g}$ to $g\left(z^{\prime}\right)$ at the Bragg scale, equation (29) becomes

$$
\begin{aligned}
S\left(k_{\text {Bragg }}\right) & \sim \frac{1}{\pi h}\left|\int_{-h / 2}^{h / 2} \frac{\tilde{n}_{e, \text { Bragg }}}{n_{e 0}} \frac{e^{i k_{\text {Bragg }} z^{\prime}}+e^{-i k_{\text {Bragg }} z^{\prime}}}{2} \exp \left(i k_{\text {Bragg }} z^{\prime}\right) d z^{\prime}\right|^{2} \\
& \sim \frac{h}{4 \pi}\left(\frac{\tilde{n}_{e, B \text { Bragg }}}{n_{e 0}}\right)^{2} .
\end{aligned}
$$

[39] Equation (26) metal can be expressed in terms of a volume reflectivity $\eta_{R}=n \sigma_{\text {eff }}$ where $n$ is the number density of perfect reflectors each having an effective cross section $\sigma_{\text {eff. }}$. For a plane wave incident on a layer of thickness $h$ the fractional reflected power would then be

$$
R=\eta_{R} h
$$


Combining equation (26) metal with equation (32) gives the volume reflectivity in terms of the spectral power at the Bragg wavelength.

$$
\begin{aligned}
\eta_{R} & =\frac{\pi}{4 c^{2}}\left(\frac{4 \pi n_{e, \text { metal }} n_{d}(0) r_{d}^{2} e^{2} d}{m_{e} \varepsilon_{0} v_{\text {metal }}}\right)^{2} S\left(k_{\text {Bragg }}\right) \\
& =\frac{16 \pi^{4}}{c^{2}}\left(\pi n_{d}(0) r_{d}^{2} \frac{\left(f_{\text {pe }}^{\text {metal }}\right)^{2}}{v_{\text {metal }}}\right)^{2}\left(\frac{\tilde{n}_{e, \text { Bragg }}}{n_{e 0}}\right)^{2} h d^{2} .
\end{aligned}
$$

[40] Using $f_{p e}^{\text {metal }}=1.6 \times 10^{15} \mathrm{~Hz}, \pi r_{d}^{2} n_{d}(0)=2 \times 10^{-6} \mathrm{~m}^{-1}$ from Raizada et al. [2007], $v_{\text {metal }}=1.8 \times 10^{13} \mathrm{~s}^{-1}, h=10^{3} \mathrm{~m}$, $\tilde{n}_{e, \text { Bragg }} / n_{e 0} \sim 10^{-4}$ from Blix et al. [2003], and assuming $d=$ $0.1 \mathrm{~nm}$ gives $\eta_{R} \sim 10^{-16} \mathrm{~m}^{-1}$; using $d=3 \mathrm{~nm}$ gives $\eta_{R} \sim 10^{-13} \mathrm{~m}^{-1}$. These estimates of volume reflectivity are consistent with observed volume reflectivity values $\eta_{R} \sim$ $10^{-16}-10^{-13} \mathrm{~m}^{-1}$ reported by Kirkwood et al. [2007].

[41] Finally, it is important to emphasize that because our model predicts that a radar signal reflects from the conduction electrons in a metal film coating the dust grains and not from free electrons or from electron holes, our model is consistent with Rapp et al.'s [2003a] observation that radar reflection occurs at bite-out locations (i.e., where there no free electrons, see Figure 2 of Rapp et al. [2003a]). In contrast, a model based on reflection from electron holes (spatial irregularities in electron depletion that are anticorrelated with dust grain density) could not explain reflection from bite-out locations.

\section{Monolayer Formation Time}

[42] The proposed model is credible only if the flux of Fe/ $\mathrm{Na}$ atoms on an ice grain is sufficiently large to form a metal film in a reasonable time. We now calculate this formation time by taking into account the detailed behavior of $\mathrm{Fe} / \mathrm{Na}$ atoms migrating from top to bottom of the mesospheric $\mathrm{Fe} /$ $\mathrm{Na}$ layers and then to a noctilucent cloud immediately below the $\mathrm{Fe} / \mathrm{Na}$ layers.

[43] The instantaneous meteoroid input flux will have some dependence on longitude and latitude because of the Earth's rotation. We define the average atomic Fe/ $\mathrm{Na}$ flux over the Earth's surface area $4 \pi R_{E}^{2}$ as

$$
\langle\Gamma\rangle=\frac{1}{4 \pi R_{E}^{2}} \int d s \Gamma
$$

and this average will be independent of rotation. Assuming a 30 ton per day meteoroid input having $0.6 \% \mathrm{Na}$ content gives $\langle\Gamma\rangle \simeq 10^{8} \mathrm{~m}^{-2} \mathrm{~s}^{-1}$ for Na; Plane [2004] gives the $\mathrm{Fe}$ flux as also being $\langle\Gamma\rangle \simeq 10^{8} \mathrm{~m}^{-2} \mathrm{~s}^{-1}$. The average atomic $\mathrm{Fe} / \mathrm{Na}$ vertical flux can be written in terms of an altitudedependent mean atomic $\mathrm{Na}$ density $n(z)$ and an altitudedependent mean velocity $u(z)$ in which case $\langle\Gamma\rangle=n(z) u(z)$. The $\mathrm{Fe} / \mathrm{Na}$ layers thus occur where $u(z)$ is very small so $n(z)$ becomes very large.

[44] Satellite measurements [Fan et al., 2007] show that $n(z)$ at polar latitudes has a strong seasonal behavior indicating that the northern and southern hemispheres must be considered as separate isolated systems. As shown schematically in Figure 3, the northern hemisphere $\mathrm{Fe} / \mathrm{Na}$ layers can be modeled as a confinement vessel for atomic $\mathrm{Fe} / \mathrm{Na}$ with height $H$ and area $A_{h}$ such that the equilibrium leak rate of $\mathrm{Fe} / \mathrm{Na}$ atoms at the top is balanced by the exhaust of $\mathrm{Fe} / \mathrm{Na}$ atoms at an equal rate into a sink at the bottom. The northern hemisphere winter $\mathrm{Na}$ density is $n=$ $6 \times 10^{9} \mathrm{~m}^{-3}$ [Fan et al., 2007] indicating $u=\langle\Gamma\rangle / n=1.7 \times$ $10^{-2} \mathrm{~m} \mathrm{~s}^{-1}$ in the Na layer. The southern hemisphere winter Fe density is $n=2 \times 10^{10} \mathrm{~m}^{-3}$ [Plane et al., 2004], i.e., about three times higher than the $\mathrm{Na}$ winter density. Since the $\mathrm{Fe} / \mathrm{Na}$ layer winter vertical extent is $H=10^{4} \mathrm{~m}$ [Plane et al., 2004; Fan et al., 2007], the residence time of a Na atom in a metal layer is $t=H / u \sim 7$ days; in summer $H$ is somewhat smaller. Fe would have a somewhat similar residence time. Because the residence time is multi-day, $\mathrm{Fe}$ or $\mathrm{Na}$ atoms will move substantial horizontal distances in the prevailing winds and this horizontal motion will smear out any diurnal dependence of the input flux. This is consistent with the observation that the polar $\mathrm{Na}$ layer has negligible diurnal variation [Plane, 2004]. Since the extraterrestrial input flux has a 5:1 diurnal variation [Plane, 2004] the polar Na layer's lack of diurnal variation implies that a $\mathrm{Na}$ atom entering the top of the layer will meander over the entire hemisphere on migrating to the bottom of the layer. Presumably Fe would behave in a similar fashion.

[45] The density and profile of polar $\mathrm{Fe}$ and $\mathrm{Na}$ layers change drastically when noctilucent clouds are present [Plane et al., 2004; Thayer and Pan, 2006; Fan et al., 2007]. There is a sharp cutoff of the bottom of the Na layer and the $\mathrm{Na}$ density is reduced from its winter value of $n_{\mathrm{Na}}=$ $6 \times 10^{9} \mathrm{~m}^{-3}$ to become just $n=10^{9} \mathrm{~m}^{-3}$ in summer [Fan et al., 2007], i.e., an $\sim 85 \%$ reduction in density. Similarly, there is a sharp cutoff of the bottom of the southern hemisphere $\mathrm{Fe}$ layer and the $\mathrm{Fe}$ density is reduced from its late February value of $n_{\mathrm{Fe}}=2 \times 10^{10} \mathrm{~m}^{-3}$ to become just $n=3 \times 10^{9} \mathrm{~m}^{-3}$ in January when noctilucent clouds are present [Plane et al., 2004]. This also corresponds to an $\sim 85 \%$ reduction in density. Raizada et al. [2007] have reported a similar reduction for potassium atoms. Plane et al. and Fan et al. attribute these observed $85 \%$ reductions in $\mathrm{Fe}$ and $\mathrm{Na}$ layer densities from winter to summer despite the same input flux $\langle\Gamma\rangle$ as due to a complete uptake of Fe and $\mathrm{Na}$ atoms by noctilucent cloud ice grains; i.e., the cloud acts as a near-perfect sink for $\mathrm{Fe}$ and $\mathrm{Na}$ atoms.

[46] The noctilucent cloud can thus be modeled as a cryopump having sufficient pumping speed to lower the $\mathrm{Fe}$ and $\mathrm{Na}$ densities by $85 \%$ compared to the density when noctilucent clouds are not present. The pumping speed can be calculated using standard cryopump analysis and taking into account that the average velocity of $150 \mathrm{~K} \mathrm{Na}$ atoms is $v_{N a}=(8 \kappa T / \pi m)^{1 / 2}=370 \mathrm{~m} / \mathrm{s}$ while the average velocity of $\mathrm{Fe}$ atoms is $v_{\mathrm{Fe}}=240 \mathrm{~m} / \mathrm{s}$. The flux of $\mathrm{Fe}$ or $\mathrm{Na}$ atoms crossing an imaginary plane from one side to the other is $n v / 4$ giving a cryopumping speed $S=v A / 4$ where $A$ is the pump surface area [O'Hanlon, 1980]. Equilibrating the $\mathrm{Na}$ input with the pumping by the noctilucent cloud "cryopump" sink gives

$$
\langle\Gamma\rangle A_{h}=\frac{n_{N a} v_{N a} A_{\text {cloud }}}{4}
$$

where $A_{h}$ is the northern hemisphere area and $A_{\text {cloud }}$ is the cloud "cryopump" surface area (see Figure 3 ). Using the 
summer Na layer density $n_{N a}=10^{9} \mathrm{~m}^{-3}$ [Fan et al., 2007] gives

$$
\frac{A_{\text {cloud }}}{A_{h}}=\frac{4\langle\Gamma\rangle}{n_{N a} v_{N a}}=10^{-3}
$$

which is not inconsistent with a 11 June 2007 AIM spacecraft noctilucent cloud image indicating that $A_{\text {cloud }}$ is about $20 \%$ of the area of Greenland.

[47] Repeating the calculation for Fe using $n_{\mathrm{Fe}}=3 \times 10^{9} \mathrm{~m}^{-3}$ [Plane et al., 2004] and $v_{F e}=240 \mathrm{~m} / \mathrm{s}$ gives $A_{\text {cloud }} / A_{h}=0.6 \times$ $10^{-3}$ which is similar to the Na estimate.

[48] The collisional mean free path of atoms with background mesospheric air molecules is $l_{m f p} \sim 1 \mathrm{~cm}$. This means that the actual distance traveled by an $\mathrm{Fe}$ or $\mathrm{Na}$ atom as it migrates from top to bottom of a noctilucent cloud greatly exceeds the cloud height $h$. Since the Fe or $\mathrm{Na}$ atom follows a random walk as sketched in Figure $3, h^{2}=$ $\mathcal{N}_{\text {col }} l_{m f p}^{2}$ where $\mathcal{N}_{\text {col }}$ is the number of collisions the Fe or $\mathrm{Na}$ atom undergoes to make a displacement $h$. The length of the actual zig-zag path traveled by the atom is $\mathcal{L}=\mathcal{N}_{\text {col }} l_{m f p}$ $=h^{2} / l_{m f p}$ so using $h=10^{3} \mathrm{~m}$, the atom will have traveled $\mathcal{L}$ $\sim 10^{8} \mathrm{~m}$. The probability that the atom hits an ice grain having cross-section $\pi r_{d}^{2}$ when the ice grains have density $n_{d}$ is $P=1-\exp \left(-n_{d} \pi r_{d}^{2} \mathcal{L}\right)$. The observations presented in Figure 5d of Raizada et al. [2007] give $\pi r_{d}^{2} n_{d}=2 \times 10^{-6}$ $\mathrm{m}^{-1}$ in which case $n_{d} \pi r_{d}^{2} \mathcal{L} \sim 2 \times 10^{2}$. Thus $\mathrm{Fe}$ or $\mathrm{Na}$ atoms have essentially unity probability of striking ice grains even though the cloud optical depth is just $n_{d} h \pi r_{d}^{2}=2 \times 10^{-3}$.

[49] Since the noctilucent cloud acts as a near-perfect sink for all downward $\mathrm{Fe}$ or $\mathrm{Na}$ atoms incident on it and since the cloud is the dominant summer-time pumping mechanism, the number of $\mathrm{Fe}$ and $\mathrm{Na}$ atoms per second trapped by the cloud is $\left(\left\langle\Gamma_{N a}\right\rangle+\left\langle\Gamma_{F e}\right\rangle\right) A_{h}$ in steady state. Because the number of ice grains in a cloud is $n_{d} h A_{\text {cloud }}$, the number of metal atoms deposited per second on each ice grain is

$$
\dot{N}_{d}=\frac{\left\langle\Gamma_{N a}\right\rangle+\left\langle\Gamma_{F e}\right\rangle}{n_{d} h} \frac{A_{h}}{A_{\text {cloud }}} .
$$

Using equation (36) this becomes

$$
\dot{N}_{d} \simeq \frac{n_{N a} v_{N a}}{2 n_{d} h} .
$$

[50] The number of metal atoms in a mono-layer on the surface of dust grain having radius $r_{d}$ is

$$
N_{\text {monolayer }}=4 \pi r_{d}^{2} n_{e, \text { metal }}^{2 / 3}
$$

The time to form a monolayer is thus

$$
t_{\text {mono }}=\frac{N_{\text {monolayer }}}{\dot{N}_{d}}=\frac{8 \pi n_{d} r_{d}^{2} h n_{e, \text { metal }}^{2 / 3}}{n_{N a} v_{N a}} \simeq 4 \text { days }
$$

using $\pi n_{d} r_{d}^{2}=2 \times 10^{-6} \mathrm{~m}^{-1}$ [Raizada et al., 2007], $h=10^{3} \mathrm{~m}$, summer-time Na layer density $n=10^{9} \mathrm{~m}^{-3}$ [Fan et al., 2007], summer-time Fe layer density $n \sim 3 \times 10^{9} \mathrm{~m}^{-3}, n_{N a} v_{N a} \sim$ $n_{\mathrm{Fe}} v_{\mathrm{Fe}} \sim 4 \times 10^{11} \mathrm{~m}^{-2} \mathrm{~s}^{-1}$ and $n_{e, \text { metal }}=2.5 \times 10^{28} \mathrm{~m}^{-3}$. Thus $0.1 \mathrm{~nm}$ of metal would be deposited on an ice grain in 33 hours.
[51] At the beginning of the summer season, the actual deposition time would be much shorter, because the $\mathrm{Na}$ and Fe layers would then each start with about six times the densities assumed in the above calculation. The beginning of the season dynamics can be estimated by calculating the inventory of $\mathrm{Na}$ and $\mathrm{Fe}$ atoms in their respective layers before cloud formation and comparing this to the number of atoms required to coat the noctilucent cloud dust grains with the assumed metallic film. Using $R_{E}=6.3 \times 10^{6} \mathrm{~m}$ and assuming equation (36) still holds, it is seen that $A_{\text {cloud }}=$ $10^{-3} \times 2 \pi R_{E}^{2}=2.5 \times 10^{11} \mathrm{~m}^{2}$. The number of metal atoms required to coat all the dust grains in a noctilucent cloud having height $h$, area $A_{\text {cloud }}$ and dust density $n_{d}$ with a metal film of thickness $d$ is

$$
N_{\text {coating }}=n_{d} h A_{\text {cloud }} \times 4 \pi r_{d}^{2} d n_{\text {metal }} .
$$

Using $\pi r_{d}^{2} n_{d}=2 \times 10^{-6} \mathrm{~m}^{-1}, h=10^{3} \mathrm{~m}, n_{\text {metal }}=2.5 \times$ $10^{28} \mathrm{~m}^{-3}$ gives

$$
N_{\text {coating }}=5 \times 10^{37} d
$$

so $N_{\text {coating }}=5 \times 10^{28}$ atoms if $d=1 \mathrm{~nm}$.

[52] Using the late February Fe value of $n_{\mathrm{Fe}}=2 \times 10^{10}$ $\mathrm{m}^{-3}$ in Plane et al. [2004], the number of Fe atoms in a hemispheric metal layer is

$$
N_{\text {layer }}=2 \pi R_{E}^{2} H n_{F e}=5 \times 10^{28}
$$

and the number of $\mathrm{Na}$ atoms will be about one third as many. Thus the inventory of atoms in the Fe and Na layers is sufficient to provide a $1 \mathrm{~nm}$ coating which is enough for the metal film electrons to dominate free electrons for an $r_{d}=10 \mathrm{~nm}$ ice grain and to dominate by a factor of 10 for a $100 \mathrm{~nm}$ ice grain. The depletion of the hemispheric metal layer inventory would thus be minimal for coatings up to a few tenths of nanometers and so these wintertime $\mathrm{Fe}$ and $\mathrm{Na}$ densities should be used for calculating the incident fluxes for the initial coatings. Thus the first $0.1 \mathrm{~nm}$ would take about 5 hours to deposit with additional layers taking longer; this estimate is slower than Plane et al.'s [2004] calculation that the e-folding time for depletion of an Fe layer by a noctilucent cloud is 2 hours since Plane et al.'s calculation would predict that $90 \%$ of the metal layer would be deposited in 5 hours rather than $10 \%$. By the time the coating becomes $1 \mathrm{~nm}$ thick, most of the winter-time metal layer inventory would have been flushed out onto the ice dust grains and the next $0.1 \mathrm{~nm}$ would take 33 hours as the metal layers would now have the summer-time density which is only one sixth of the winter-time density.

[53] Thus if dust grains suddenly formed when the metal layers had their maximum densities, it should be possible to achieve in a matter of hours to days the $d=0.1-3 \mathrm{~nm}$ thick coatings required to provide the observed volumetric $10^{-16}-10^{-13} \mathrm{~m}^{-1}$ radar reflectivity at $50 \mathrm{MHz}$.

\section{References}

Blix, T. A., M. Rapp, and F. J. Lubken (2003), Relations between small scale electron number density fluctuations, radar backscatter, and charged 
aerosol particles, J. Geophys. Res., 108(D8), 8450, doi:10.1029/ 2002JD002430.

Bradshaw, F. J., and S. Pearson (1956), The electrical resistivity of sodium between 78-degrees-K and 372-degrees-K, Proc. Phys. Soc. Lond. B, 69(4), $441-448$

Cho, J. Y. N., and M. C. Kelley (1993), Polar mesosphere summer radar echoes-observations and current theories, Rev. Geophys., 31(3), 243265.

Eidhammer, T., and O. Havnes (2001), Size dependence of the mesospheric dust temperature and its influence on the noctilucent clouds and polar mesosphere summer echo phenomena, J. Geophys. Res., 106(A11), 24,831-24,841.

Fan, Y., J. M. C. Plane, J. Gumbel, J. Stegman, and E. J. Llewellyn (2007), Satellite measurements of the global mesospheric sodium layer, Atmos. Chem. Phys., 7, 4107-4115.

Fedorov, D. V., P. Zahn, and I. Mertig (2007), Manifestation of quantum confinement in transport properties of ultrathin metallic films, Thin Solid Films, 515(17), 6921-6926.

Gardner, C. S., J. M. C. Plane, W. L. Pan, T. Vondrak, B. J. Murray, and X. Z. Chu (2005), Seasonal variations of the $\mathrm{Na}$ and Fe layers at the South Pole and their implications for the chemistry and general circulation of the polar mesosphere, J. Geophys. Res., 110, D10302, doi:10.1029/ 2004JD005670.

Havnes, O., F. Melandso, C. Lahoz, T. K. Aslaksen, and T. Hartquist (1992), Charged dust in the Earth's mesopause - effects on radar backscatter, Phys. Scr., 45, 535-544.

Havnes, O., and L. I. Naesheim (2007), On the secondary charging effects and structure of mesospheric dust particles impacting on rocket probes, Ann. Geophys., 250(3), 623-637.

Havnes, O., J. Troim, T. Blix, W. Mortensen, L. I. Naesheim, E. Thrane, and T. Tonnesen (1996), First detection of charged dust particles in the Earth's mesosphere, J. Geophys. Res., 101(A5), 10,839-10,847.

Hoffner, J., and J. S. Friedman (2004), The mesospheric metal layer topside: A possible connection to meteoroids, Atmos. Chem. Phys., 4, $801-$ 808 .

Kirkwood, S., I. Wolf, H. Nilsson, P. Dalin, D. Mikhaylova, and E. Belova (2007), Polar mesosphere summer echoes at Wasa, Antarctica $\left(73^{\circ} \mathrm{S}\right)$ : First observations and comparison with $68^{\circ} \mathrm{N}$, Geophys. Res. Lett., 340 L15803, doi:10.1029/2007GL030516.

Lubken, F. J., and J. Hoffner (2004), Experimental evidence for ice particle interaction with metal atoms at the high latitude summer mesopause region, Geophys. Res. Lett., 31, L08103, doi:10.1029/2004GL019586.

McNeil, W. J., S. T. Lai, and E. Murad (1998), Differential ablation of cosmic dust and implications for the relative abundances of atmospheric metals, J. Geophys. Res., 103(D9), 10,899-10,911.

Murray, B. J., and J. M. C. Plane (2005), Uptake of Fe, Na and K atoms on low-temperature ice: Implications for metal atom scavenging in the vicinity of polar mesospheric clouds, Phys. Chem. Chem. Phys., 70(23), $3970-3979$

O'Hanlon, J. F. (1980), A User's Guide to Vacuum Technology, pp. $221-$ 226, Wiley-InterScience, Hoboken, N. J.
Plane, J. M. C. (2003), Atmospheric chemistry of meteoric metals, Chem. Rev., 103, 4963-4984.

Plane, J. M. C. (2004), A time-resolved model of the mesospheric Na layer: Constraints on the meteor input function, Atmos. Chem. Phys., 4, 627638.

Plane, J. M. C., B. J. Murray, X. Z. Chu, and C. S. Gardner (2004), Removal of meteoric iron on polar mesospheric clouds, Science, 304(5669), 426-428.

Raizada, S., M. Rapp, F. J. Lubken, J. Hoffner, M. Zecha, and J. M. C. Plane (2007), Effect of ice particles on the mesospheric potassium layer at Spitsbergen $\left(78^{\circ} \mathrm{N}\right)$, J. Geophys. Res., 112, D08307, doi:10.1029/ 2005JD006938.

Rapp, M., and F. J. Lubken (1999), Modelling of positively charged aerosols in the polar summer mesopause region, Earth Planets Space, 51, $799-807$.

Rapp, M., and F. J. Lubken (2001), Modelling of particle charging in the polar summer mesosphere. Part I: General results, J. Atmos. Sol. Terr. Phys., 63(8), 759-770.

Rapp, M., and F. J. Lubken (2004), Polar mesosphere summer echoes (PMSE): Review of observations and current understanding, Atmos. Chem. Phys, 4, 2601-2633.

Rapp, M., F. J. Lubken, and T. A. Blix (2003a), Small scale density variations of electrons and charged particles in the vicinity of polar mesosphere summer echoes, Atmos. Chem. Phys., 3, 1399-1407.

Rapp, M., F. J. Lubken, P. Hoffmann, R. Latteck, G. Baumgarten, and T. A. Blix (2003b), PMSE dependence on aerosol charge number density and aerosol size, J. Geophys. Res., 1080(D8), 8441, doi:10.1029/ 2002JD002650

Smiley, B., M. Rapp, T. A. Blix, S. Robertson, M. Horanyi, R. Latteck, and J. Fiedler (2006), Charge and size distribution of mesospheric aerosol particles measured inside NLC and PMSE during MIDAS MaCWAVE 2002, J. Atmos. Sol. Terr. Phys., 68(1), 114-123.

Thayer, J. P., and W. L. Pan (2006), Lidar observations of sodium density depletions in the presence of polar mesospheric clouds, J. Atmos. Sol. Terr. Phys., 68(1), 85-92.

Vondrak, T., J. M. C. Plane, and S. R. Meech (2006a), Influence of submonolayer sodium adsorption on the photoemission of the $\mathrm{Cu}(111) /$ water ice surface, J. Chem. Phys., 125(22), 224702, doi:10.163/1.2397675.

Vondrak, T., J. M. C. Plane, and S. R. Meech (2006b), Photoemission from sodium on ice: A mechanism for positive and negative charge coexistence in the mesosphere, J. Phys. Chem., B, 110(9), 3860-3863.

Wood, R. W. (1933), Remarkable optical properties of the alkali metals, Phys. Rev., 44, 353-360.

Yakshinskiy, B. V., and T. E. Madey (2001), Electron- and photonstimulated desorption of $\mathrm{K}$ from ice surfaces, J. Geophys. Res., 106(E12), 33,303-33,307.

P. M. Bellan, Applied Physics, Caltech, 1200 E. California Blvd., Pasadena, CA 91125, USA. (pbellan@its.caltech.edu) 